\title{
Congenital web of the common bile duct combined with multiple intrahepatic duct stricture: a case report of successful radiological intervention
}

\author{
Hanseul Lim ${ }^{1}$, Shin Hwang ${ }^{1}$, Gi-Young Ko ${ }^{2}$, Hyejin Han $^{1}$ \\ ${ }^{1}$ Department of Surgery, Asan Medical Center, University of Ulsan College of Medicine, Seoul, Korea \\ ${ }^{2}$ Department of Radiology, Asan Medical Center, University of Ulsan College of Medicine, Seoul, Korea
}

\begin{abstract}
Congenital web formations are extremely rare anomalies of the extrahepatic biliary tree. We herein report a case of common bile duct septum combined with multiple intrahepatic bile duct strictures in a 74-year-old female patient who was successfully treated with radiological intervention. The patient initially visited the hospital because of upper abdominal pain. Imaging studies revealed multifocal strictures with dilatation in both intra- and extrahepatic ducts; the final clinical diagnosis was congenital common bile duct web combined with multiple intrahepatic duct strictures. Surgical treatment was not indicated because multiple biliary strictures were untreatable, and the disease was clinically diagnosed as benign. The multiple strictures were extensively dilated twice through bilateral percutaneous transhepatic biliary drainage (PTBD) for 2 months. After 1 month of observation, PTBD catheters were successfully removed. The patient is doing well at 6 months after completion of the radiological intervention, with the maintenance of normal liver function. Congenital web of the bile duct is very rare, and its treatment may vary depending on the patterns of biliary stenosis. In cases where surgical intervention is not indicated for congenital web and its associated disease, radiological intervention with balloon dilatation can be a viable therapeutic option.
\end{abstract}

Keywords: Bile duct obstruction; Congenital anomaly; External drainage; Interventional radiology; Obstructive jaundice

\section{Introduction}

The incidence of congenital anomalies of the extrahepatic biliary system is approximately $10 \%$ [1]. The vast number of these anomalies are asymptomatic, and they may be diagnosed as incidentally identified findings during cholecystectomy or living liver donor work-up $[2,3]$. Congenital web formations are extremely rare anomalies of the extrahepatic biliary tree. The clinical manifestations of congenital webs or strictures of the extrahepatic ducts include obstructive jaundice, dilatation of the proximal bile ducts, or spontaneous rupture of the extrahepatic biliary system. A considerable proportion of patients with congenital webs might live without any recognizable symptoms for a long period, probably due to partial biliary obstruction. They can be diagnosed with a congenital web of the extrahepatic biliary tree in adulthood, with the exclusion of other known causes of acquired stricture or web formation. Herein, we report a case of common bile duct (CBD) septum combined with multiple intrahepatic bile duct strictures in a 74-year-old female patient who was successfully treated with radiological intervention.

Received: June 1, 2021 • Revised: June 12, 2021 • Accepted: June 12, 2021

Corresponding author: Shin Hwang, MD, PhD, FACS

Department of Surgery, Asan Medical Center, University of Ulsan College of Medicine, Olympic-ro 43-gil 88, Songpa-gu, Seoul 05505, Korea

Tel: +82-2-3010-3930• Fax: +82-2-3010-6701•E-mail: shwang@amc.seoul.kr 


\section{Case}

Ethical statements: This study was approved by the Institutional Review Board (IRB) of Asan Medical Center (IRB No: 2021-0683), and informed consent was obtained from the patient.

A 74-year-old female patient was referred to our institution with a diagnosis of hilar bile duct stenosis. The patient was admitted to a local hospital because of upper abdominal pain. At that time, aspartate transaminase (AST), alanine transaminase (ALT), total bilirubin, and cancer antigen 19-9 (CA 19-9) were elevated to 1,870 IU/ $\mathrm{mL}, 1,390 \mathrm{IU} / \mathrm{mL}, 1.4 \mathrm{mg} / \mathrm{dL}$, and $177 \mathrm{U} / \mathrm{mL}$, respectively. Abdominal computed tomography (CT) showed diffuse dilatation of the intra- and extrahepatic bile ducts (Fig. 1A, 1B). Magnetic reso- nance cholangiopancreatography (MRCP) showed multifocal biliary webs without an anomalous pancreaticobiliary junction (Fig. $1 C, 1 D)$. Passage of the $\mathrm{CBD}$ web through endoscopic retrograde cholangiopancreatography had failed, so a left percutaneous transhepatic biliary drainage (PTBD) catheter was inserted. Brush cytology of the CBD showed mild, nonspecific chronic inflammation.

After the liver transaminase levels were normalized, the patient was admitted to our institution for further evaluation and treatment. MRCP performed at our institution showed multifocal stricture with dilatation in both intra- and extrahepatic ducts, suggesting that the stricture was more likely to be benign than malignant (Fig. 2). The serum CA 19-9 level was reduced to $22 \mathrm{U} / \mathrm{mL}$. Fluorodeoxyglucose positron emission tomography-CT showed no significant hypermetabolic activity, suggesting a primary lesion in the hepatobiliary system, thereby suggesting that a benign CBD stricture was more likely than a low metabolic malignant stricture.
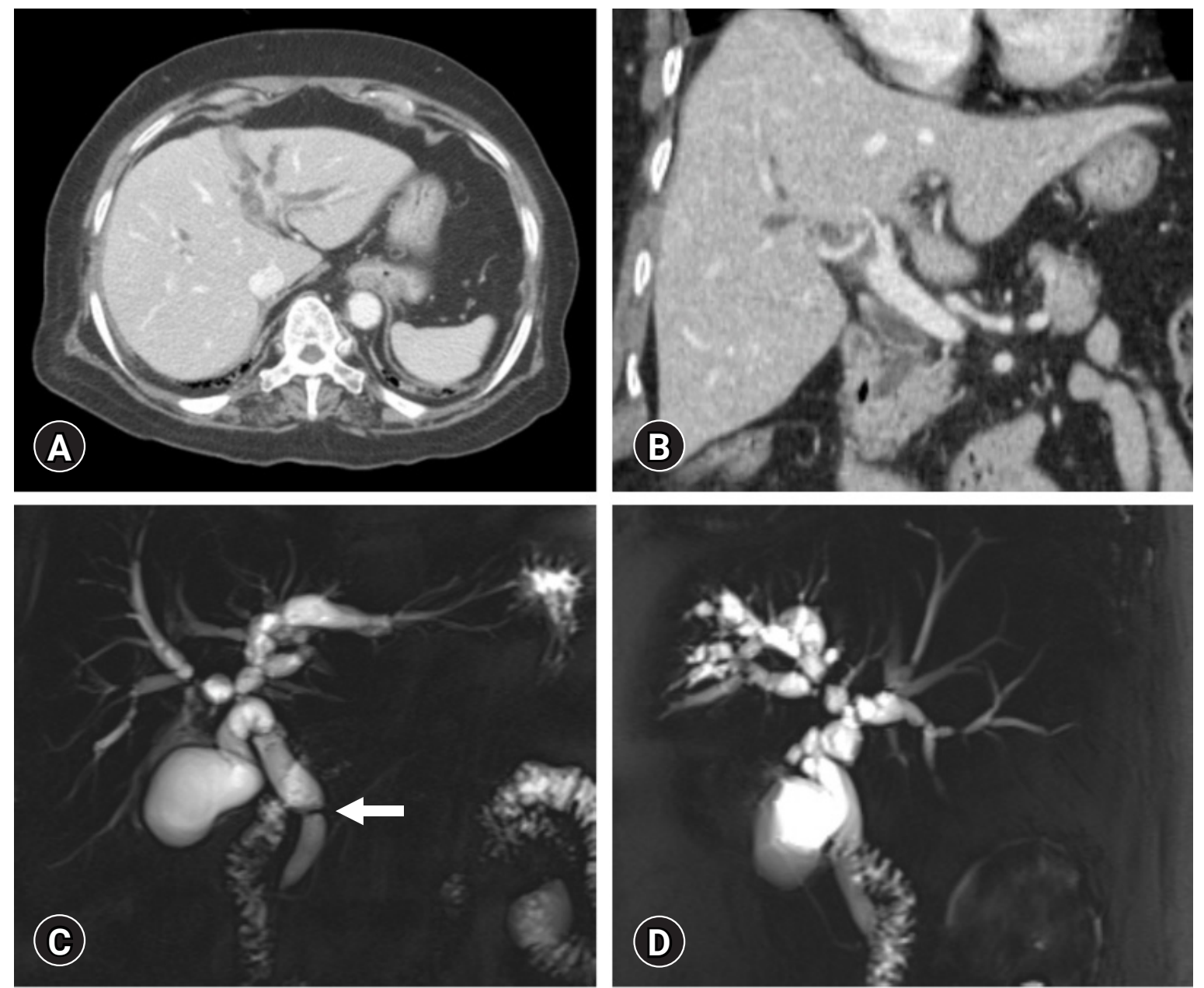

Fig. 1. Initial imaging studies. (A, B) Abdominal computed tomography shows diffuse dilatation of the intra- and extrahepatic bile ducts. (C, D) Magnetic resonance cholangiopancreatography shows multifocal biliary webs without anomalous pancreaticobiliary junction. Arrow indicates the location of a common bile duct web. 

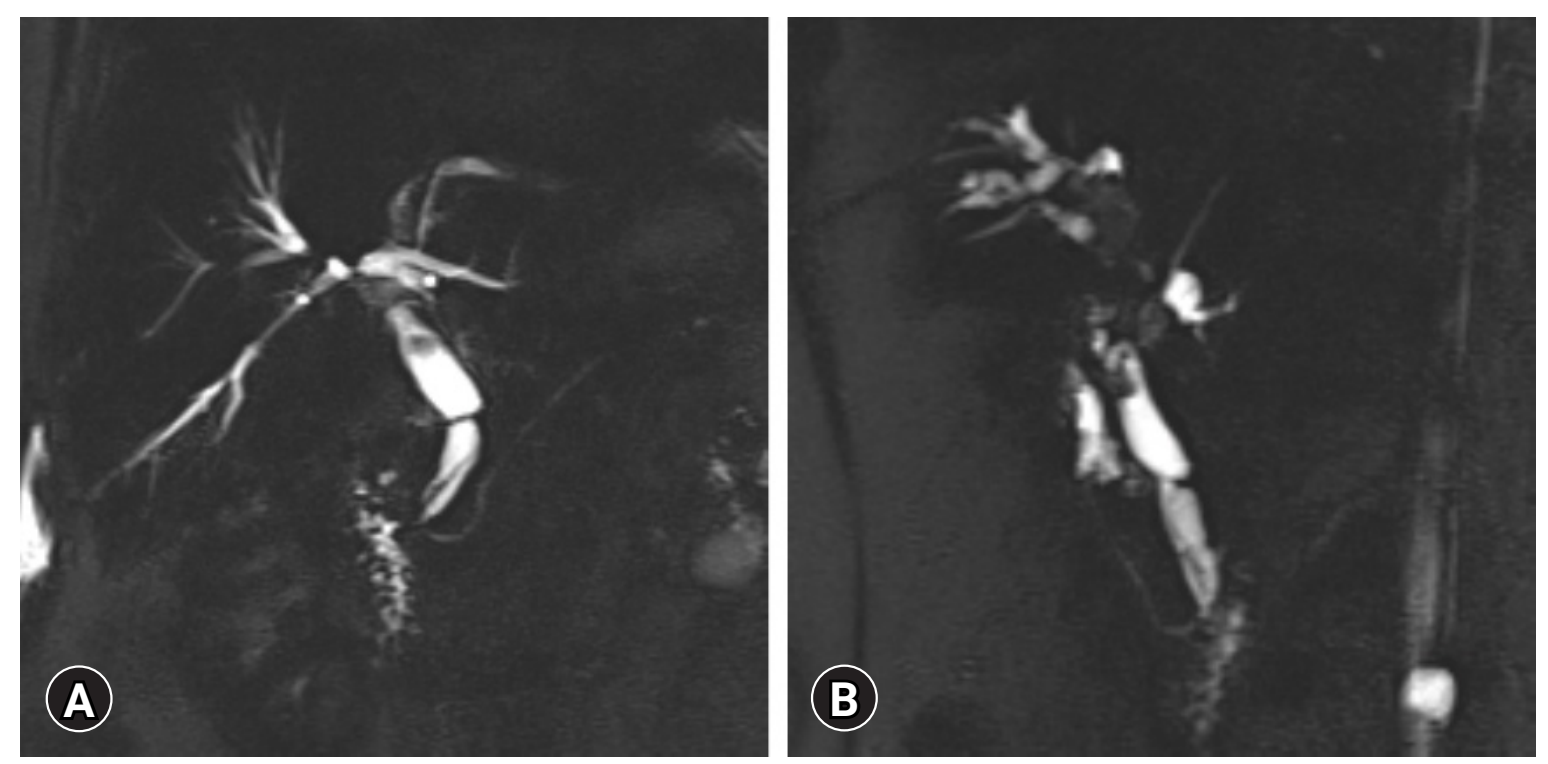

Fig. 2. The second magnetic resonance cholangiopancreatography shows multifocal stricture with dilatation in both intra- and extrahepatic ducts, suggesting that the stricture is more likely to be benign than malignant. The (A) location and (B) degree of the multifocal strictures are visualized.
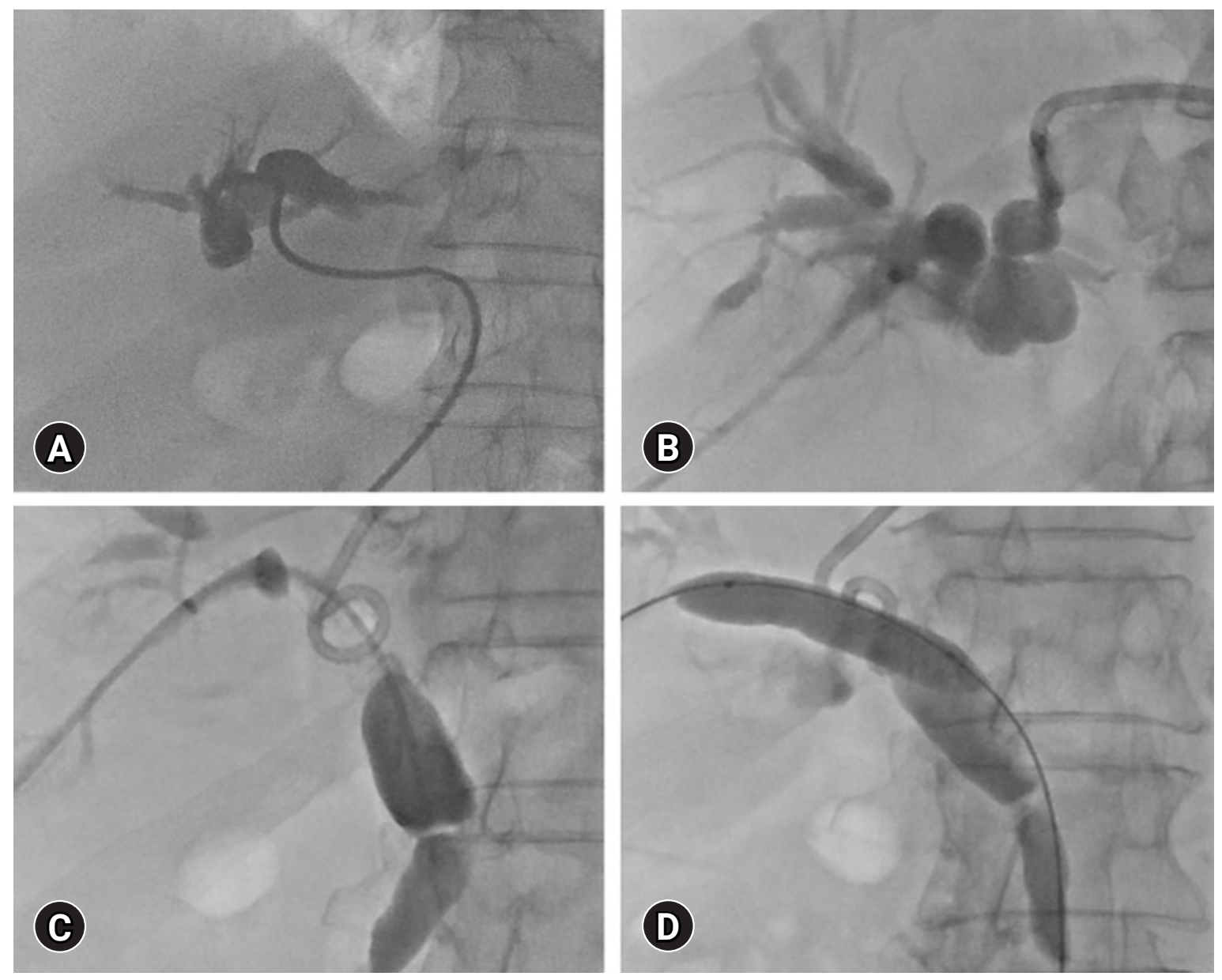

Fig. 3. Direct cholangiography findings. (A) The left-sided tubogram shows complete occlusion of the left hepatic duct. (B) The right-sided tubogram shows bile duct occlusion at the hepatic hilum. (C, D) The common bile duct web is finally cannulated after repeated trials, and then balloon dilatation of the intra- and extrahepatic stricture is performed. 

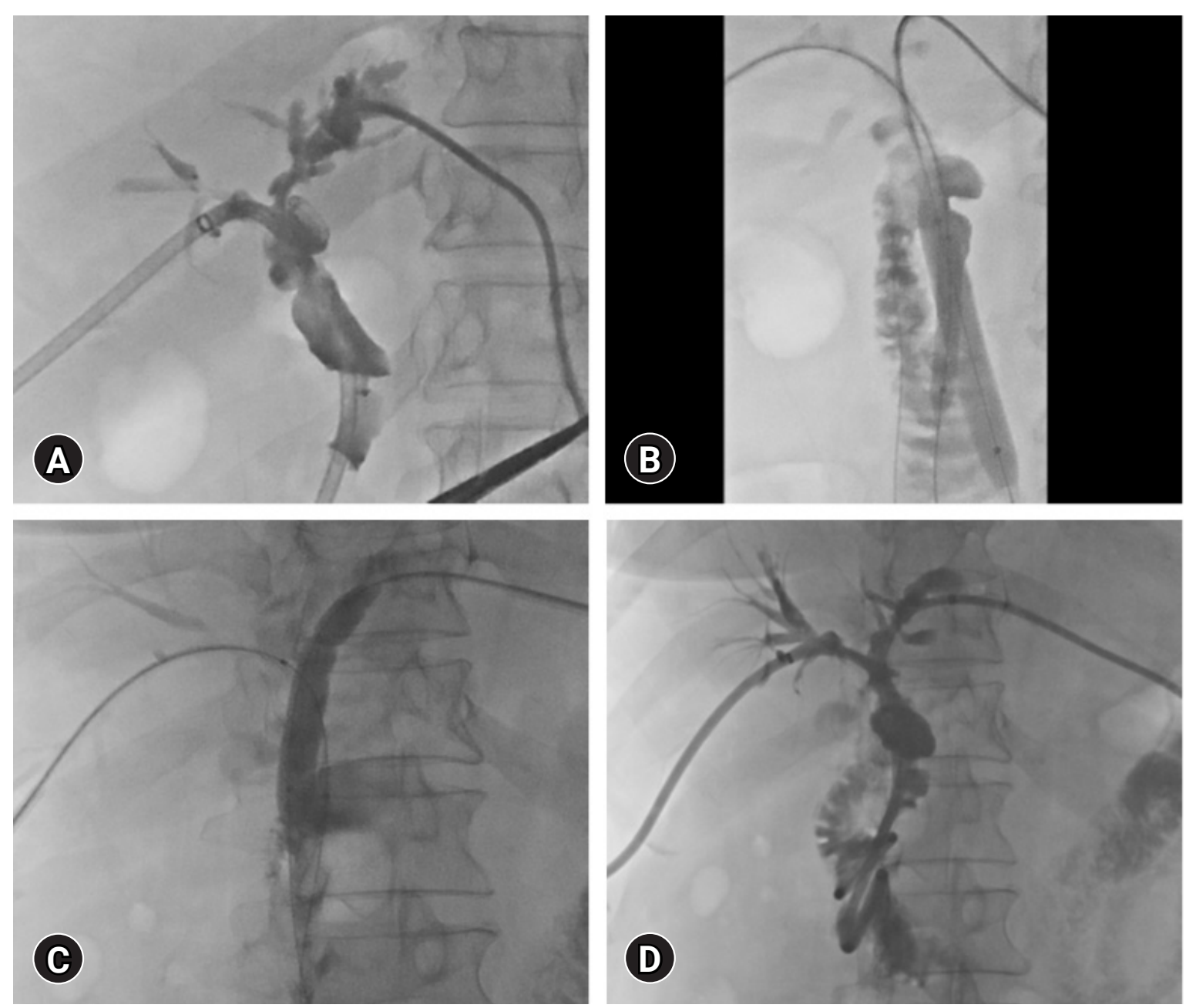

Fig. 4. The second balloon dilatation of the biliary stricture. (A) Tubogram shows the presence of residual strictures. $(B, C)$ The second balloon dilatation of the intra- and extrahepatic stricture is extensively performed. (D) Tubogram shows improvement in multiple strictures.

The clinical diagnosis was ultimately determined to be congenital CBD web combined with multiple intrahepatic duct strictures.

The treatment plan was wait-and-see following balloon dilatation of the strictures. A tubogram through the left PTBD showed complete occlusion of the left hepatic duct (Fig. 3A). A right PTBD catheter was inserted and passed across the occlusion area at the hepatic hilum but was unable to cross the CBD web (Fig. $3 B)$. After 4 days, the CBD web was finally penetrated by the guidewire, after which balloon dilatation of the intra- and extrahepatic strictures was conducted (Fig. 3C, 3D). The patient was then discharged with clamping of the two PTBD catheters and readmitted 1 month later. At this point, second extensive balloon dilatation of the intra- and extrahepatic strictures was performed through the right and left PTBD (Fig. 4). The patient was discharged again with clamping of the two PTBD catheters and readmitted 1 month later. A follow-up tubogram showed the good passage of the bile duct (Fig. 5). The two PTBD tubes were removed sequentially with close monitoring of liver function. The liver function test remained normal after PTBD removal, and hepatobiliary scintigraphy showed a 90-minute excretion rate of $82 \%$ without significant obstruction.

The patient has been doing well for 6 months after completion of the radiological intervention, with a 90-minute excretion rate of $80 \%$ on follow-up hepatobiliary scintigraphy (Fig. 6). Follow-up CT showed a thin doughnut-shaped web at the distal bile duct (Fig. 7). The patient was administered ursodeoxycholic acid (UDCA) to facilitate biliary drainage.

\section{Discussion}

The incidence of congenital webs of the extrahepatic ducts is reported to be very low, with approximately 20 cases being reported 


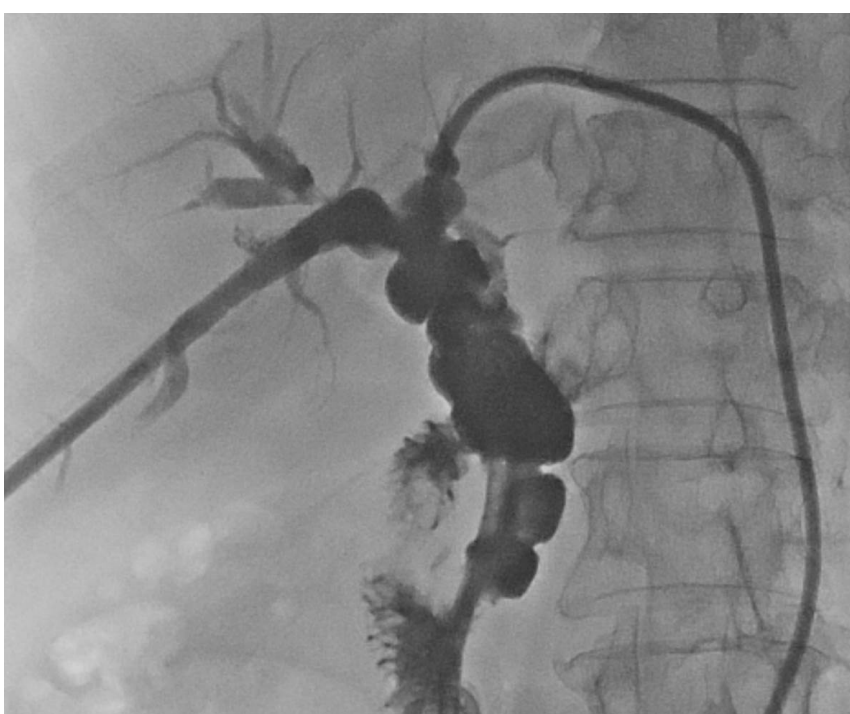

Fig. 5. Follow-up tubogram showing the good passage of the bile duct.
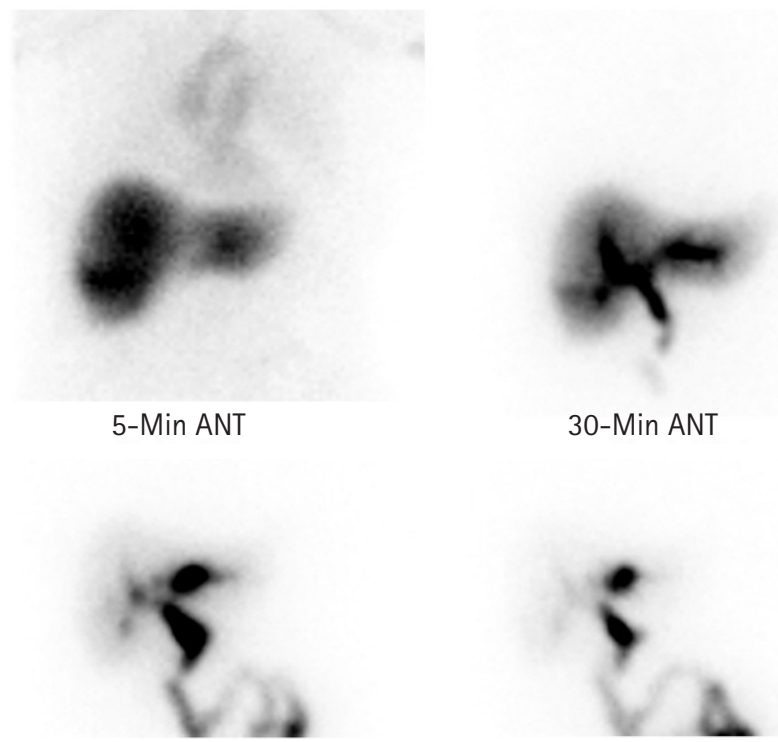

60-Min ANT
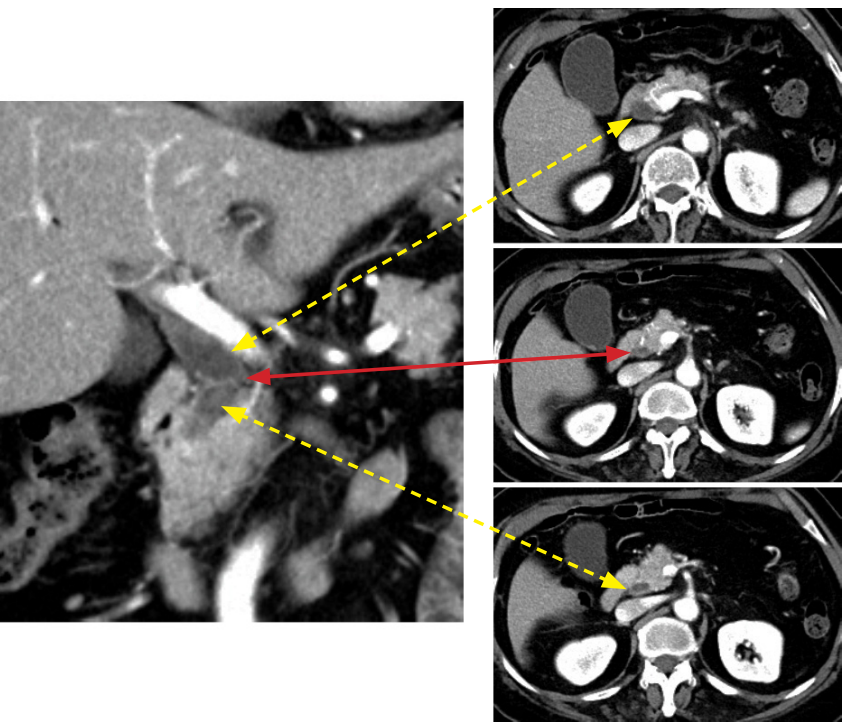

Fig. 7. Follow-up computed tomography taken at 6 months after the treatment showing a thin doughnut-shaped web at the distal bile duct. Dotted arrows indicate the proximal and distal levels of the bile duct to the web. Solid arrow indicates the level of a web at the distal bile duct.

tal webs of the biliary system $[4,6]$.

It is important to differentiate congenital webs from other causes of biliary stenosis. Biliary stenosis of iatrogenic causes is usually located in the CBD or right hepatic duct. Multiple intrahepatic and extrahepatic stenoses are often present in patients with primary sclerosing cholangitis $[11,12]$. Isolated biliary strictures are also known to be associated with blunt trauma to the abdomen, radiation therapy of the upper abdomen $[13,14]$, and localized sclerosing cholangitis [11].

The physiological implication of a web of the biliary system may not be identical to the stenoses of the extrahepatic duct associated with other causes. Although the biliary web can induce symptomatic biliary obstruction, biliary drainage from the liver may remain undisturbed in the majority of patients. Patients may initially be asymptomatic or present with vague and nonspecific symptoms, such as abdominal pain, nausea, and vomiting. Early in the disease process, patients may only demonstrate elevations of transaminase and alkaline phosphatase levels along with ductal dilatation, without obstructive jaundice. Our patient also showed elevated liver enzymes and ductal dilatation without manifestation of obstructive jaundice. In clinical practice, overt obstructive jaundice occurs only after near-complete CBD obstruction, regardless of the cause of $\mathrm{CBD}$ obstruction.

A completely developed septum-inducing total biliary obstruction usually presents with obstructive jaundice soon after birth. If not treated effectively, this disease can lead to secondary biliary cir- 
rhosis or even spontaneous perforation of the biliary system [15]. Delayed development of symptoms in adulthood is usually associated with incompletely formed or perforated webs, which cause only partial biliary obstruction. Our patient remained asymptomatic for over 70 years, likely because the biliary obstruction caused by the web was incomplete. The patient presented with multifocal biliary webs at the intrahepatic and extrahepatic bile ducts. Considering that the majority of the intrahepatic ducts were dilated, especially proximal to each biliary stricture, we presumed that multiple congenital webs had induced partial biliary obstruction for a long period. Finally, some symptoms of biliary obstruction occurred due to progressive deterioration of biliary drainage that was associated with the focal stricture-induced bottleneck phenomenon.

The majority of congenital biliary webs are associated with cholelithiasis or choledocholithiasis [9]. The association between congenital web and gallstone formation has not been clearly demonstrated. Congenital web-induced partial biliary obstruction can favor gallstone formation. However, an increased incidence of gallstone disease can lead to the diagnosis of asymptomatic webs that would not be diagnosed if gallstone disease is absent.

While standard imaging methods such as ultrasonography and CT may reveal bile duct dilatation, they are unlikely to reveal the presence of a biliary web. MRCP is useful for delineating the locations and shapes of the biliary webs. Congenital webs are usually not associated with malignancy. However, it is reasonable to resect this structure and obtain a frozen section biopsy prior to completing the procedure [10]. If a web is encountered during exploration of the extrahepatic biliary tree, it is necessary to perform an intraoperative cholangiogram to assess the rest of the ductal system to prevent inadvertent damage due to the association of these webs with other coexisting biliary anomalies. Surgical treatment was not indicated for our patient because of the presence of combined multiple biliary strictures, and the disease was clinically diagnosed as benign.

In a Japanese multicenter randomized trial that compared the CBD stone recurrence rate after bile duct stone removal, UDCA administration was shown to be an effective treatment for preventing CBD stone recurrence [16]. In a Chinese randomized clinical trial with liver transplant patients, UDCA treatment decreased the levels of serum ALT and AST during the 4 weeks after transplantation, and it also decreased the incidence of biliary sludge and casts within the first year. However, UDCA administration did not affect the overall outcomes up to 5 years after transplantation [17]. Although there is still some debate about its preventive effect on gallstone formation, we suggest the administration of UDCA in patients with a high risk of choledocholithiasis.

Congenital webs at the bile duct are very rare, and their treatment may vary depending on the patterns of biliary stenosis. Our experience suggests that radiological intervention with balloon dilatation can be a viable therapeutic option if surgical intervention is not indicated for congenital web and its associated disease.

\section{Notes}

\section{Conflicts of interest}

No potential conflict of interest relevant to this article was reported.

\section{Funding}

None.

\section{Author contributions}

Conceptualization: SH; Data curation: HL, HH; Methodology, Visualization: SH, GYK; Writing-original draft: SH, HL; Writing-review \& editing: all authros.

\section{ORCID}

Hanseul Lim, https://orcid.org/0000-0002-6128-855X

Shin Hwang, https://orcid.org/0000-0002-9045-2531

Gi-Young Ko, https://orcid.org/0000-0003-4617-1799

Hyejin Han, https://orcid.org/0000-0002-6091-1717

\section{References}

1. Lamah M, Dickson GH. Congenital anatomical abnormalities of the extrahepatic biliary duct: a personal audit. Surg Radiol Anat 1999;21:325-7.

2. Choi SH, Kim KW, Kwon HJ, Kim SY, Kwon JH, Song GW, et al. Clinical usefulness of gadoxetic acid-enhanced MRI for evaluating biliary anatomy in living donor liver transplantation. Eur Radiol 2019;29:6508-18.

3. Takeishi K, Shirabe K, Yoshida Y, Tsutsui Y, Kurihara T, Kimura $\mathrm{K}$, et al. Correlation between portal vein anatomy and bile duct variation in 407 living liver donors. Am J Transplant 2015;15: $155-60$.

4. Furukawa H, Hara T, Taniguchi T. A case of septum formation of the common hepatic duct combined with an anomalous hepatic duct of the caudate lobe. Gastroenterol Jpn 1992;27:1027.

5. Kato S, Nakagawa T, Kobayashi H, Arai E, Isetani K. Septum formation of the common hepatic duct associated with an anomalous junction of the pancreaticobiliary ductal system and 
gallbladder cancer: report of a case. Surg Today 1994;24:534-7.

6. Dolar ME, Ates KB, Dalay AR, Caner ME, Sasmaz N, Sahin B. Congenital stricture of the common hepatic duct due to a web: an unusual case without jaundice. Hepatogastroenterology 1993;40:194-5.

7. Fisher MM, Chen SH, Dekker A. Congenital diaphragm of the common hepatic duct. Gastroenterology 1968;54:605-10.

8. Kottoor R, Alvares JF, Devarbhavi H. Successful endoscopic therapy of an obstructing common bile duct web. Gastrointest Endosc 2001;53:126-8.

9. Papaziogas B, Lazaridis C, Pavlidis T, Galanis I, Paraskevas G, Papaziogas T. Congenital web of the common bile duct in association with cholelithiasis. J Hepatobiliary Pancreat Surg 2002; 9:271-3.

10. Margolis M, Schein M. Biliary web: a rare cause of extrahepatic biliary obstruction. Dig Surg 2001;18:317-9.

11. Gulliver DJ, Baker ME, Putnam W, Baillie J, Rice R, Cotton PB. Bile duct diverticula and webs: nonspecific cholangiographic features of primary sclerosing cholangitis. Am J Roentgenol 1991;157:281-5.

12. Panés J, Bordas JM, Bruguera M, Cortés M, Rodés J. Localized sclerosing cholangitis? Endoscopy 1985;17:121-2.

13. Yoon KH, Ha HK, Kim MH, Seo DW, Kim CG, Bang SW, et al. Biliary stricture caused by blunt abdominal trauma: clinical and radiologic features in five patients. Radiology 1998;207:73741.

14. Cherqui D, Palazzo L, Piedbois P, Charlotte F, Duvoux C, Duron JJ, et al. Common bile duct stricture as a late complication of upper abdominal radiotherapy. J Hepatol 1994;20:6937.

15. Shih SL, Lin JC, Lee HC, Blickman JG. Unusual causes of obstructive jaundice in children: diagnosis on CT. Pediatr Radiol $1992 ; 22: 512-4$.

16. Yamamoto R, Tazuma S, Kanno K, Igarashi Y, Inui K, Ohara H, et al. Ursodeoxycholic acid after bile duct stone removal and risk factors for recurrence: a randomized trial. J Hepatobiliary Pancreat Sci 2016;23:132-6.

17. Wang SY, Tang HM, Chen GQ, Xu JM, Zhong L, Wang ZW, et al. Effect of ursodeoxycholic acid administration after liver transplantation on serum liver tests and biliary complications: a randomized clinical trial. Digestion 2012;86:208-17. 